\title{
Specialized Vascularization of the Primate Visual Cortex
}

\author{
Dake Zheng, Anthony-Samuel LaMantia, and Dale Purves \\ Department of Neurobiology, Duke University Medical Center, Durham, North Carolina 27710
}

\begin{abstract}
We have analyzed blood vessel distribution in the primary and secondary visual cortices of the squirrel monkey in relation to cortical modules, laminae, and cytoarchitectonic areas. Measurements of microvessel length in tangential sections through the primary visual cortex showed that blobs are more richly vascularized than intervening cortical regions. Thus, the mean total length of microvessel profiles per unit was $42 \%$ greater within these cortical modules than within adjacent (interblob) areas. Total microvessel length per unit area in another class of module, the stripes In the secondary visual cortex, was $27 \%$ greater than in interstripe regions. Microvessel distribution also varied systematically from layer to layer in the primary visual cortex, being greatest in lamina IVc. Finally, the overall microvessel length per unit area in sections of the primary visual cortex was $26 \%$ greater than that in the secondary visual cortex. These observations indicate that the modular, laminar, and regional organization of the primate visual cortex is reflected in the underlying distribution of cortical microvessels. These vascular patterns should be discernable in living animals with vascular contrast agents and appropriate imaging techniques.
\end{abstract}

The primate visual cortex has a characteristic modular, laminar, and regional organization (Van Essen, 1979; Hendrickson, 1985). In the present study, we have asked whether the cortical microvasculature is arranged in patterns that correspond to these structural and functional features of the visual cortex. The answer to this question is relevant to two issues. First, vascular patterns corresponding to these cortical features might provide additional insight into the nature of such organization. Second, distinctive vascular patterns might provide a way of visualizing these functionally important subdivisions in the brains of intact animals.

The most obvious regional and laminar distinctions in the primate visual cortex are those between the primary and secondary visual cortices; these cytoarchitectonic differences create an obvious boundary between area 17 and area 18 in the monkey. Both these cortical regions comprise a variety of discrete functional units called modules (Livingstone and Hubel, 1984; Hendrickson, 1985; Hubel, 1986, 1988). Among the most striking of these units are the cytochrome oxidase-positive blobs in area 17 and the stripes in area 18 (see Fig. 1). Blobs have been found in the primary visual cortex of all primates examined to date (Horton and IIubel, 1981; Carroll and Wong-Riley,

\footnotetext{
Received Jan. 22, 1991; revised Mar. 22, 1991; accepted Apr. 5, 1991.

We thank Ann Richards, Mary Beth Groelle, and Feri Zsuppan for valuable assistance and David Fitzpatrick, Allan Johnson, Larry Katz, and David Riddle for helpful comments on the manuscript.

Correspondence should be addressed to Dale Purves at the above address.

Copyright (C) 1991 Society for Neuroscience $0270-6474 / 91 / 112622-08 \$ 03.00 / 0$
}

1984; Horton, 1984) and project specifically to a subset of the stripes in area 18 (Hubel and Livingstone, 1987; Livingstone and Hubcl, 1987).

In the course of studying the development of blobs in the monkey visual cortex (Purves and LaMantia, 1990), we noticed that microvessels seemed more abundant within these processing units than in the intervening (interblob) regions. Because specialized vascularization might represent a distinguishing feature of modules, laminae, and visual cortical areas, we undertook a quantitative analysis of microvessel distribution in this region of the primate brain.

\section{Materials and Methods}

A total of four squirrel monkeys (Saimiri sciureus, adult females) were sedated with ketamine $(10 \mathrm{mg} / \mathrm{kg})$ and deeply anesthetized with sodium pentobarbital $(100 \mathrm{mg} / \mathrm{kg})$. They were then perfused with saline followed by a solution of $2.5 \%$ paraformaldehyde, $1.5 \%$ glutaraldehyde, and $4 \%$ sucrose in sodium phosphate buffer (Wong-Riley, 1979; Carroll and Wong-Riley, 1984). Two of these monkeys were used for analyses of vascular patterns in the tangential plane; in the other two, vascularization was assessed in the coronal plane. For analysis of vessels in the tangential plane, the opercular and calcarine cortices of the primary visual area, together with the adjoining extrastriate cortex, were dissected away from the underlying white matter and divided into several blocks. For analysis of vessels in the orthogonal plane, the occipital lobe was divided into three or four blocks for coronal sectioning. The cortical blocks were mounted flat onto glass coverslips and sectioned at a thickness of $50 \mu \mathrm{m}$ in a vibratome. The sections were stained free-floating for cytochrome oxidase to reveal blobs and stripes (Fig. 1) or cortical layers and areal borders (Fig. 2). Sections were mounted in glycerol to retain hydration (thus optimally preserving microvessel architecture) and examined by transillumination using cross-polarized light. The contrast of vessels was enhanced by stopping down the condenser aperture; the contrast of cytochrome oxidase-positive features was enhanced by illumination with blue light (CB12 filter; 400-500-nm transmittance).

To examine blood vessel distribution in blobs and stripes, 10 samples of a standard circular field measuring $1.0 \mathrm{~mm}$ in diameter from sections through layers II-III were analyzed in each animal at a final magnification of $110 \times$ (Fig. 3). In each sample, all vascular profiles in the field were traced using a camera lucida. The vast majority of blood vessels in these sections were capillaries. In order to avoid the problem of distinguishing between capillaries and larger vessels, however, every vessel in the section was traced as a single line corresponding to its length (see Fig. 3). Because the vessels in the visual cortex are very similar in size (see Figs. 3-6), and because all measurements were made at relatively low power, we concluded that overall length per 

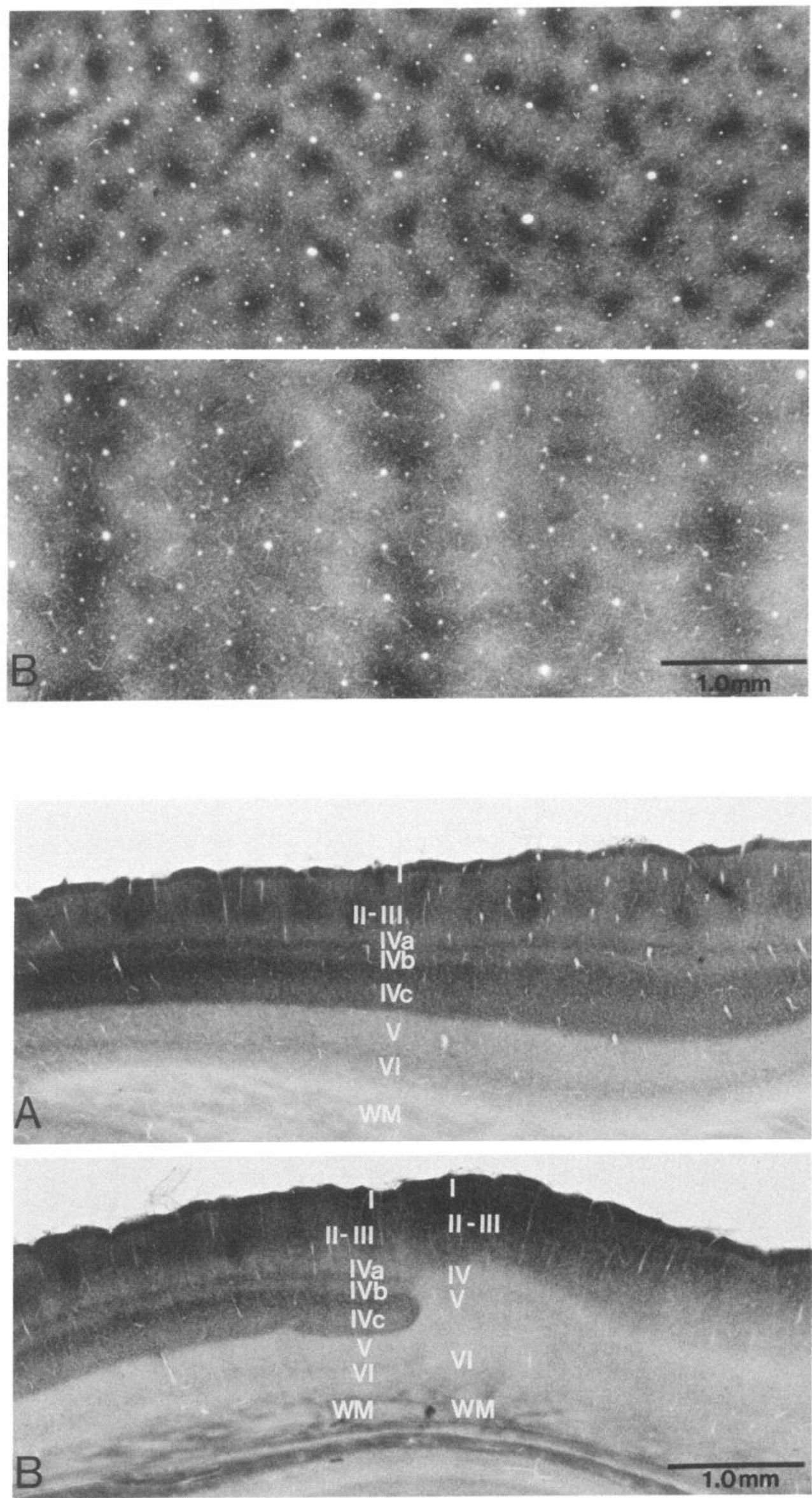

Figure 1. Low-power photomicrographs of cytochrome oxidase-stained modules in the squirrel monkey visual cortex. $A$, Blobs in a tangential section through laminae II-III of area 17. $B$, Stripes in a tangential section through laminae II-III of area 18. In some regions of the section, blobs and stripe boundaries are less clearly defined because of a tendency for bridging between adjacent blobs and variations in the intensity of staining along the length of individual stripes. Such areas were avoided in selecting regions in which to measure microvessel length.

Figure 2. Low-power photomicrographs of laminar and regional features of the squirrel monkey visual cortex stained with cytochrome oxidase. $A$, Cortical laminae in a coronal section through area 17. Note blobs in layers II and III. $B$, Border of area 17 (left) and area 18 (right). $W M$, white matter. 

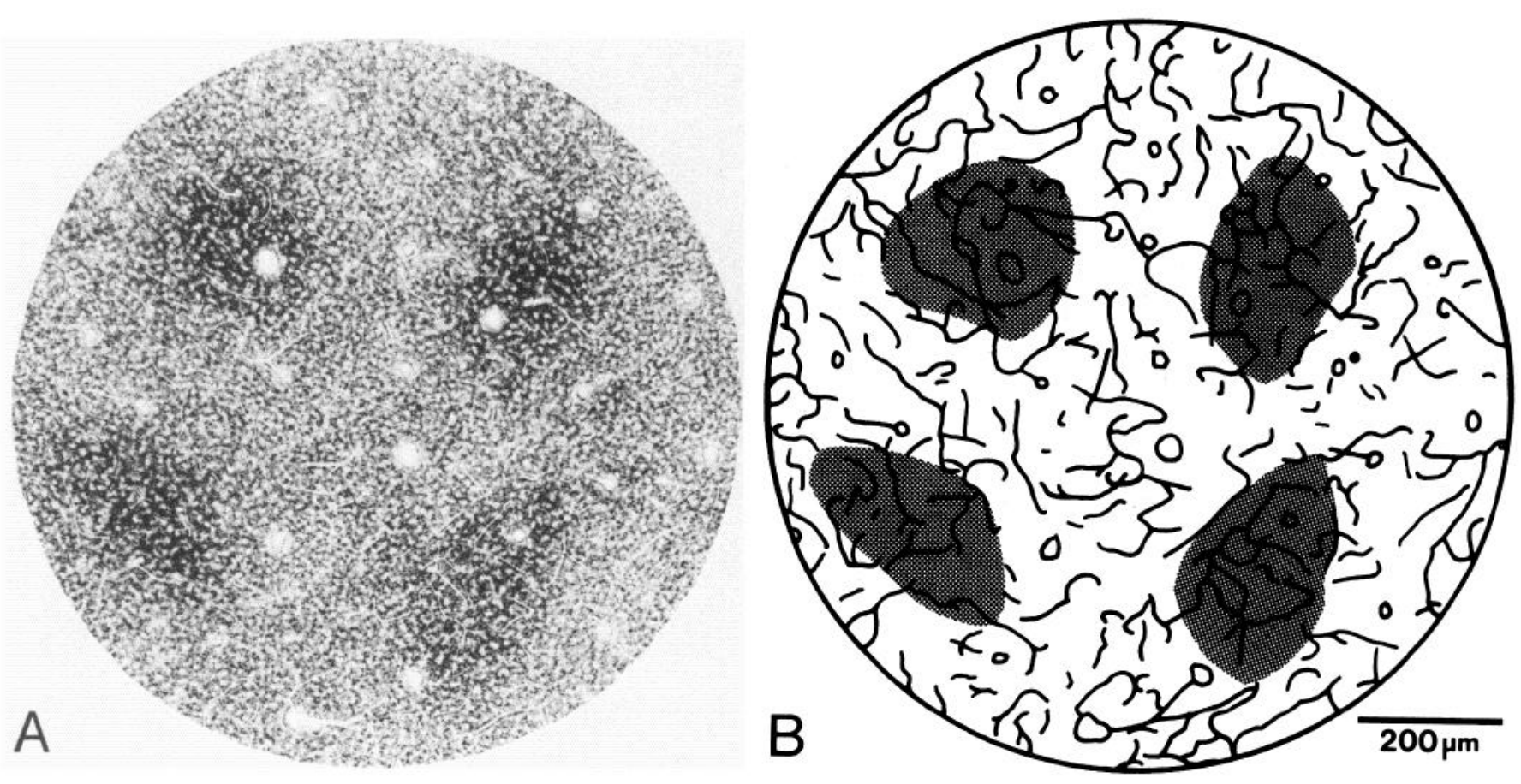

Figure 3. Measurement of microvessel distribution in blobs and interblobs. A, Higher-power view of a cytochrome oxidase-stained tangential section showing a 1.0-mm-diameter field containing four blobs. $B$, Camera lucida tracing of microvessels and blobs in the field shown in $A$. In this particular field, the total microvessel length per unit area within blobs was $23.6 \mathrm{~mm} / \mathrm{mm}^{2}$, while in the interblob region the corresponding value was $16.7 \mathrm{~mm} / \mathrm{mm}^{2}$. This difference between blobs and interblobs $(41.3 \%)$ approximates the mean value of all the samples examined (see Table 1$)$.
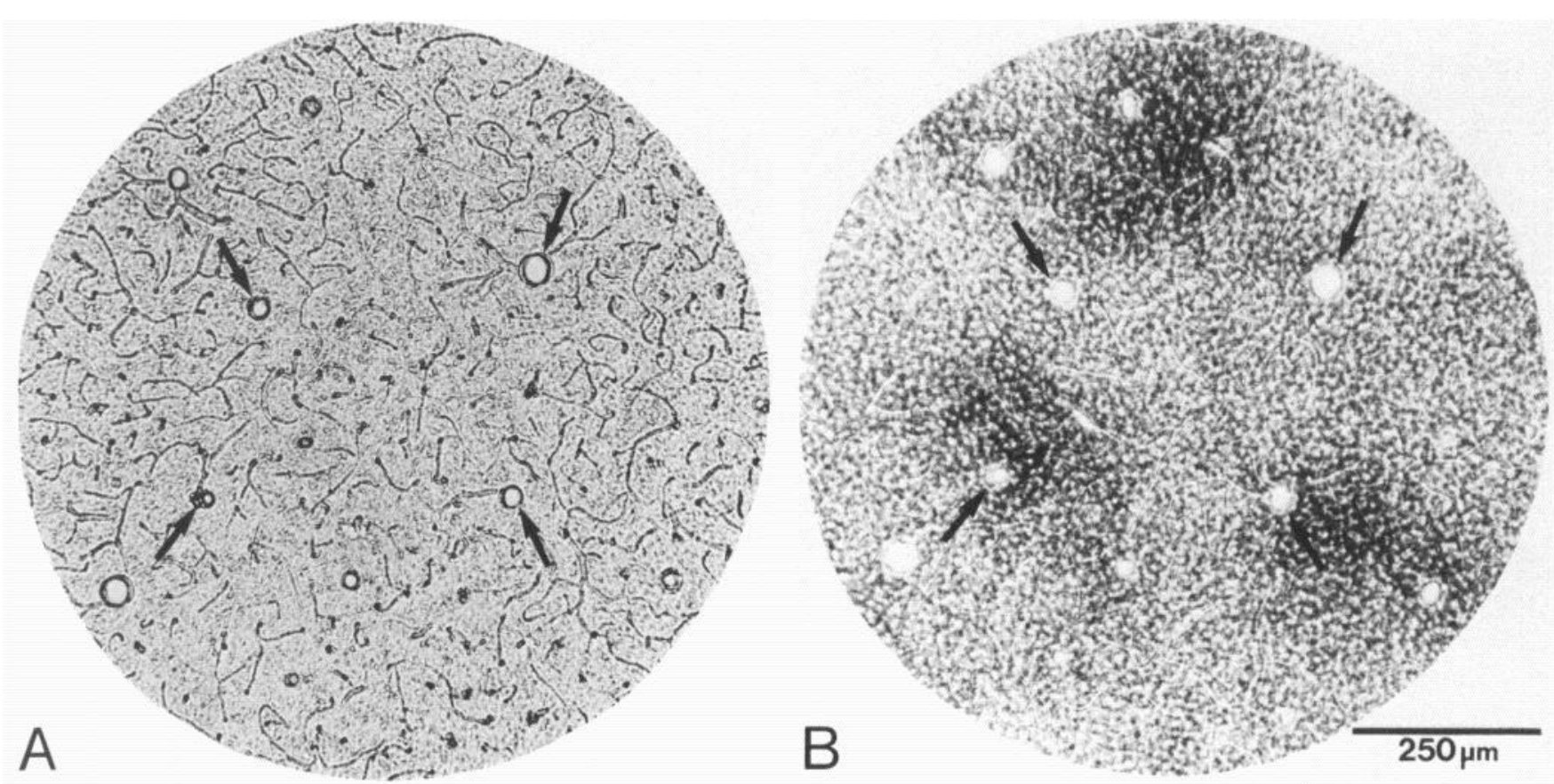

Figure 4. Control assessment of microvessel distribution in unstained sections. A, Photomicrograph of microvessels in a 1.0 -mm-diameter field from an unstained tangential section of area 17. $B$, The same field from an adjacent section stained with cytochrome oxidase. Superimposing $B$ upon $A$ allowed post hoc analysis of blood vessel distribution in blobs and interblobs; this approach reduced the possibility of experimental bias or artifact arising from background contrast. Arrows indicate corresponding radial vessels used for aligning the two sections. 
Table 1. Measurements of microvessel length per unit area in standard samples of blobs and interblobs in area 17 from two adult female squirrel monkeys

\begin{tabular}{llll} 
& \multicolumn{2}{l}{ Length/area $\left(\mathrm{mm} / \mathrm{mm}^{2}\right)$} & \\
\cline { 3 - 4 } Sample & Blobs & $\begin{array}{l}\text { Interblob } \\
\text { regions }\end{array}$ & $\begin{array}{l}\text { \% Differ- } \\
\text { ence }\end{array}$ \\
\hline
\end{tabular}

\begin{tabular}{clll}
\hline Animal 1 & & & \\
1 & 34.3 & 27.7 & 23.8 \\
2 & 32.3 & 26.0 & 24.2 \\
3 & 29.1 & 20.9 & 39.2 \\
4 & 35.8 & 21.9 & 63.5 \\
5 & 25.5 & 21.8 & 17.0 \\
6 & 23.6 & 17.3 & 36.4 \\
7 & 29.9 & 20.6 & 45.1 \\
8 & 29.6 & 19.9 & 48.7 \\
9 & 36.8 & 24.1 & 52.7 \\
10 & 28.9 & 19.6 & 47.4 \\
Mean \pm SEM & $30.6 \pm 1.3$ & $22.0 \pm 1.0$ & 39.8
\end{tabular}

Animal 2

\begin{tabular}{clll}
1 & 24.6 & 15.6 & 57.7 \\
2 & 23.3 & 17.8 & 30.9 \\
3 & 26.2 & 22.7 & 15.4 \\
4 & 32.5 & 23.0 & 41.3 \\
5 & 25.1 & 16.2 & 54.9 \\
6 & 31.7 & 21.2 & 49.5 \\
7 & 32.9 & 22.0 & 49.5 \\
8 & 43.9 & 29.4 & 49.3 \\
9 & 33.7 & 23.5 & 43.4 \\
10 & 42.1 & 28.2 & 49.3 \\
Mean \pm SEM & $31.6 \pm 2.3$ & $22.0 \pm 1.5$ & 44.1 \\
\hline
\end{tabular}

Each sample represents the analysis of a circular field $1.0 \mathrm{~mm}$ in diameter within layers II-III of the mid opercular region (see Fig. 3). The average difference observed between blobs and interblobs for the entire set of samples in the two monkeys $(42 \%)$ is highly significant $(p<0.001$, independent Student's $t$ test) Percent difference $=[($ blob value - interblob value $) /$ interblob value $] \times 100$.

unit area was the best index of microvessel distribution. Even the largest vessels in the visual cortex were less than $50 \mu \mathrm{m}$ in diameter. The blobs or stripes were then outlined, and the total vessel length $/ \mathrm{mm}^{2}$ within blobs or stripes was compared to the total vessel length $/ \mathrm{mm}^{2}$ in the intervening cortex. The borders of these modules were drawn at the point where cytochrome reactivity was judged to have fallen to background levels. Because this judgement is, to a degree, subjective, we also carried out control experiments in which the determination of blob borders was made after, rather than before, measurements of microvessel length. The total vessel length $/ \mathrm{mm}^{2}$ in blobs and interblobs was measured with a digitizing tablet and a standard morphometric system (Bioquant IV, R\&M Biometrics).

The microvessel distribution in different cortical laminae and the overall microvessel density in areas 17 and 18 were analyzed in a similar way. Ten $1.0-\mathrm{mm}$-wide vertical strips from coronal sections of the primary visual cortex in each of the two animals studied were examined to determine laminar differences in $\mathrm{mi}-$ crovessel density. At the area $17 / 18$ border, $101.0-\mathrm{mm}$-wide strips from each cytoarchitectonic area in the two animals were examined to assess the overall density of blood vessels in the primary and secondary visual cortices.

\section{Results}

Blobs

Blobs in the squirrel monkey are distributed in a regular pattern throughout area 17 at a density of $6-7 / \mathrm{mm}^{2}$ (Fig. $1 A$ ). Although
Table 2. Measurements of microvessel length per unit area in standard samples of stripes and interstrips in area 18

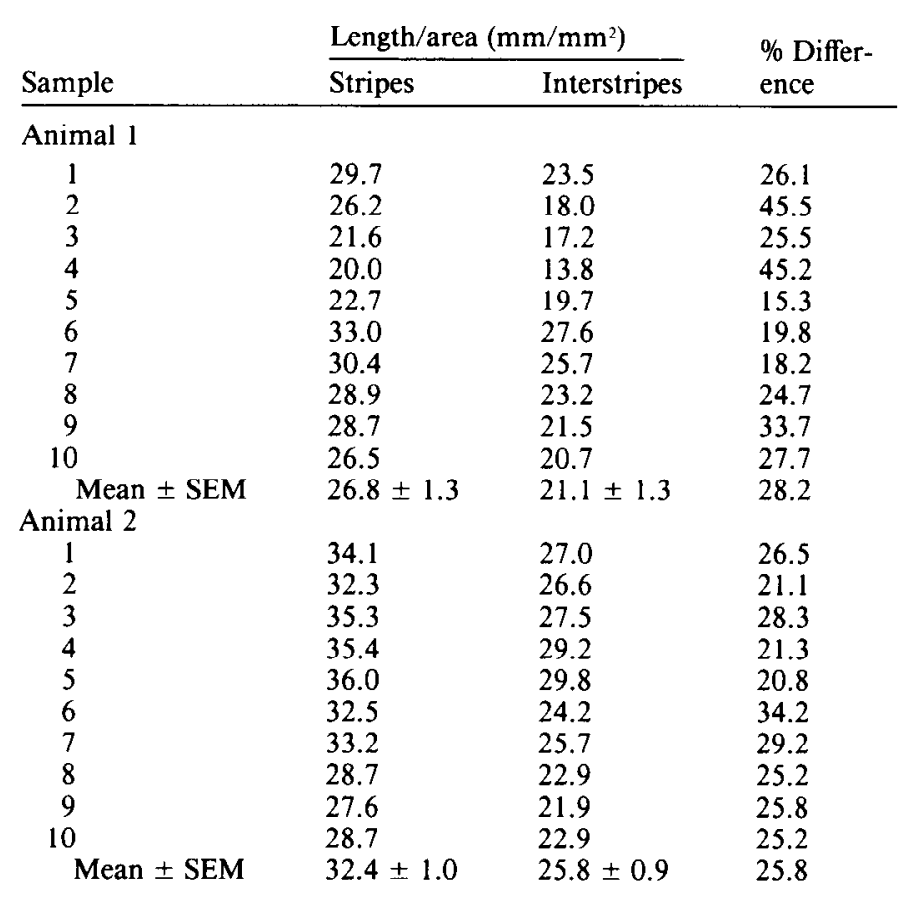

As in the analysis of blobs (Table 1), each sample represents a circular field 1.0 $\mathrm{mm}$ in diameter. The average difference observed for the entire set of samples $(27 \%)$ is again highly significant $(p<0.001$, independent Student's $t$ test).

the shape of individual blobs varies greatly, the majority are circular or ovoid in tangential sections, with an average crosssectional area of $6.2 \times 10^{4} \pm 0.2 \times 10^{4} \mu \mathrm{m}^{2}( \pm \mathrm{SEM} ; n=163)$. Figure 3 shows a representative sample of squirrel monkey cortex in which the included blobs and the associated cortical vasculature have been traced. In each of the samples analyzed in this way, the vessel density was greater inside the blobs than outside them (Table 1). On average, the overall length of $\mathrm{mi}$ crovessel profiles within blobs was $42 \%$ greater than in adjacent interblob areas.

To rule out any bias in the determination of microvessel density in blob and interblob regions, tangential sections through layers II-III were prepared and stained for cytochrome oxidase as described above; the adjacent section, however, was left unstained (Fig. 4). With only sufficient reference to the stained section to permit analysis in the appropriate region, the blood vessels in five standard fields from the unstained sections were measured as before. After the blood vessels had been traced, the position of blobs in the unstained section was determined by superimposing a tracing of the adjacent stained section onto the microvessel tracing. Large, radially oriented vessels were used for proper alignment. The microvessel density in blobs and interblobs was then determined. The results obtained in this way were similar to those from stained sections. Thus, in each of the five control samples, the density of microvessels within unstained blobs was greater than within interblobs (range, 14$46 \%$; mean, $31 \%$; cf. Table 1 ).

Finally, it was of interest to ask whether the increased density of vessels within blobs was limited to capillaries or was also 
Figure 5. Laminar differences of microvessel distribution in the primary visual cortex. A, Photomicrograph of a 1.0-mm-wide strip from a cytochrome oxidase-stained coronal section of area 17 showing cortical laminae (including the subdivisions of layer IV); note blobs $(B)$ in layers II-III. $B$, Camera lucida tracing of same field as in $A$ showing microvessel distribution. The overall length of microvessels in the different cortical laminae in this sample was (in $\mathrm{mm} / \mathrm{mm}^{2}$ ) layer I, 14.7 ; layer II-III, 30.1; layer IVa, 37.3; layer IVb, 27.5; layer IVc, 40.6; layer V, 24.8; layer VI, 27.3 ; white matter $(W M), 9.8$. These values are similar to the average values shown in Table 3.
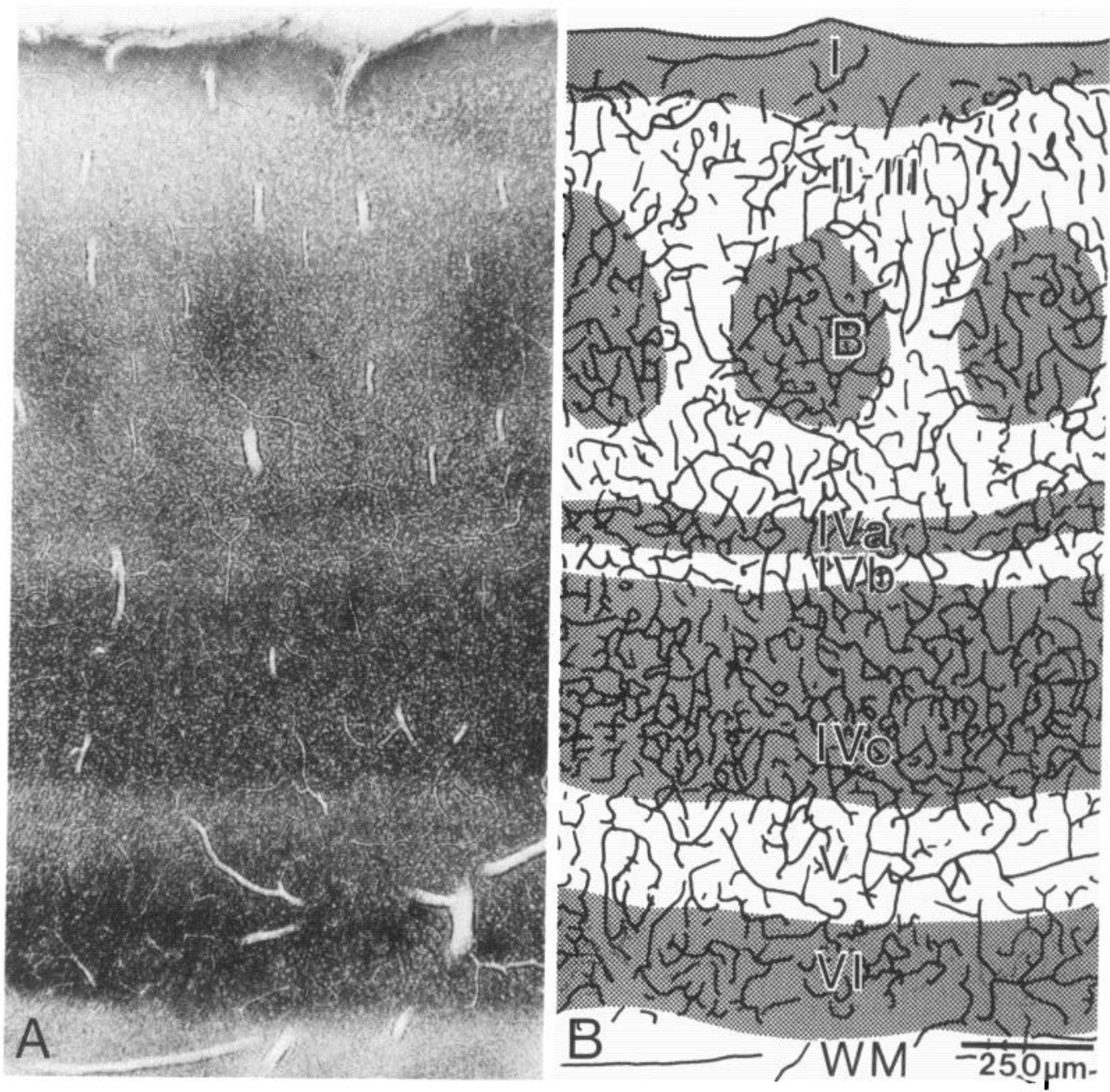

reflected in the distribution of larger vessels $(20-50 \mu \mathrm{m}$ in diameter) that penetrate radially through tangential sections (see Figs. 3, 4). Based on an analysis of 202.0 -mm-diameter circular fields in tangential sections, the average density of large radial vessels was $9.6 \pm 0.9 / \mathrm{mm}^{2}$ within blobs and $5.6 \pm 0.5 / \mathrm{mm}^{2}$ in interblob regions $( \pm \mathrm{SEM})$. Thus, the larger, radial vessels are also more prevalent within blobs than within interblobs $(p<$ 0.001 , independent Student's $t$ test).

\section{Stripes}

Similar measurements were made from stripes and interstripes that characterize area 18 (Fig. $1 B$; Horton, 1984). Because the identity of stripe subclasses by simple inspection was not always certain, no distinction was made in our analysis between thin stripes, which receive projections from blobs, and thick stripes, which do not (Livingstone and Hubel, 1987). We did, however, avoid the less heavily stained regions that occur along the length of individual stripes (see Fig. $1 B$ ). These measurements showed that cytochrome oxidase-positive stripes in area 18 are also more densely vascularized than the cytochrome oxidase-poor interstripe regions (Table 2). The mean difference in this case was $27 \%$.

\section{Laminae and areal borders}

We also examined microvessel density in coronal sections of areas 17 and 18 (Figs. 2, 5,6). The difference in vascular density measured between blobs and interblobs within layers II-III of area 17 is equally detectable in coronal sections. Microvessel density in the various laminae of the primary visual cortex differs markedly, being greatest in layer IVc and least in layer I (Table 3). In general, cytochrome oxidase reactivity and vascular density are positively correlated in the primary visual cortex of monkeys, as has been previously noted in the rat brain (Borowsky and Collins, 1989). Measurements of the overall microvessel density in the primary and secondary visual cortices showed that there is a net difference across the area $17 / 18$ border (Fig. 6). On average, the overall length of microvessel profiles per unit area in cortical strips from area 17 was $26 \%$ greater than in strips from area 18 (Table 4). This difference is readily apparent in unstained sections (Fig. 6B).

\section{Discussion}

Our analysis of blood vessels in the primate visual cortex indicates that microvessel density in the visual system is strongly related to the underlying circuitry of this region. This result is important for two reasons. First, our findings suggest that the arrangement of cortical circuits and their metabolic requirements will, as a rule, be reflected in a corresponding vascular pattern. Second, these patterns may provide a means of revealing cortical modules, layers, and areas in intact, living animals.

With respect to the first of these points, blobs and stripes in 
Table 3. Measurements of microvessel length per unit area in the various cortical laminae of area 17

Length/area $\left(\mathrm{mm} / \mathrm{mm}^{2}\right)$

\begin{tabular}{|c|c|c|c|c|c|c|c|c|}
\hline \multirow[b]{3}{*}{ Sample } & \multicolumn{8}{|c|}{ Length/area $\left(\mathrm{mm} / \mathrm{mm}^{2}\right)$} \\
\hline & \multicolumn{2}{|l|}{ Lamina } & \multirow[b]{2}{*}{ IVa } & \multirow[b]{2}{*}{ IVb } & \multirow[b]{2}{*}{ IVc } & \multirow[b]{2}{*}{$\mathrm{V}$} & \multirow[b]{2}{*}{ VI } & \multirow[b]{2}{*}{ WM } \\
\hline & $\mathrm{I}$ & II-III & & & & & & \\
\hline \multicolumn{9}{|l|}{ Animal 1} \\
\hline 1 & 17.4 & 34.9 & 29.1 & 27.0 & 41.9 & 23.0 & 27.7 & 6.7 \\
\hline 2 & 15.0 & 30.8 & 28.4 & 21.2 & 42.3 & 25.4 & 24.4 & 5.0 \\
\hline 3 & 12.4 & 22.3 & 39.4 & 21.5 & 30.6 & 25.5 & 23.2 & 8.4 \\
\hline 4 & 9.2 & 28.2 & 34.2 & 27.0 & 36.7 & 28.5 & 23.2 & 12.5 \\
\hline 5 & 9.9 & 34.2 & 37.2 & 34.1 & 41.2 & 29.2 & 24.7 & 13.4 \\
\hline 6 & 14.4 & 30.2 & 43.0 & 23.6 & 34.4 & 23.8 & 25.7 & 12.0 \\
\hline 7 & 8.2 & 26.1 & 36.9 & 27.5 & 40.3 & 20.1 & 25.1 & 13.7 \\
\hline 8 & 13.7 & 30.8 & 38.8 & 39.9 & 41.5 & 25.4 & 25.2 & 16.2 \\
\hline 9 & 8.3 & 30.3 & 29.2 & 25.2 & 39.9 & 25.7 & 26.8 & 16.1 \\
\hline 10 & 8.1 & 28.5 & 36.4 & 31.4 & 38.9 & 20.8 & 25.9 & 11.4 \\
\hline Mean \pm SEM & $11.7 \pm 1.1$ & $29.6 \pm 1.2$ & $35.3 \pm 1.6$ & $27.8 \pm 1.9$ & $38.8 \pm 1.2$ & $24.7 \pm 0.9$ & $25.2 \pm 0.5$ & $11.5 \pm 1.2$ \\
\hline \multicolumn{9}{|l|}{ Animal 2} \\
\hline 1 & 20.7 & 29.9 & 24.8 & 20.9 & 46.3 & 37.4 & 43.9 & 24.3 \\
\hline 2 & 7.9 & 33.1 & 23.2 & 28.0 & 41.9 & 32.8 & 42.1 & 27.8 \\
\hline 3 & 8.4 & 28.3 & 36.0 & 19.7 & 46.7 & 27.4 & 41.8 & 30.3 \\
\hline 4 & 10.8 & 31.4 & 39.9 & 22.7 & 41.5 & 38.5 & 43.6 & 26.1 \\
\hline 5 & 12.1 & 27.9 & 31.8 & 18.2 & 39.6 & 28.8 & 42.7 & 24.6 \\
\hline 6 & 18.7 & 28.1 & 34.7 & 32.5 & 42.1 & 36.5 & 38.4 & 19.2 \\
\hline 7 & 9.2 & 31.3 & 32.8 & 34.1 & 40.2 & 32.2 & 27.0 & 7.2 \\
\hline 8 & 13.0 & 29.5 & 52.3 & 35.4 & 43.2 & 33.2 & 37.9 & 18.8 \\
\hline 9 & 15.4 & 32.7 & 34.1 & 27.9 & 36.9 & 25.6 & 27.1 & 19.1 \\
\hline 10 & 13.1 & 31.3 & 36.3 & 26.4 & 42.7 & 31.5 & 46.1 & 12.4 \\
\hline Mean \pm SEM & $12.9 \pm 1.4$ & $30.4 \pm 0.6$ & $34.6 \pm 2.5$ & $26.6 \pm 1.9$ & $42.1 \pm 0.9$ & $32.4 \pm 1.4$ & $39.1 \pm 2.1$ & $21.0 \pm 2.3$ \\
\hline
\end{tabular}

Each sample represents the analysis of a 1.0 -mm-wide strip extending from the pial surface to the subjacent white matter (WM; see Figs. $2 A, 5$ ).

the monkey visual cortex can be seen with cytochrome oxidase, a marker of mitochondrial activity; with 2-deoxyglucose, a marker of metabolic and neuronal activity (Humphrey and Hendrickson, 1983); and with antibodies directed against neurotransmitters, neurotransmitter-synthesizing enzymes, and extracellular matrix proteins (Hendrickson et al., 1981; Fitzpatrick et al., 1987; Hendry et al., 1988). Cortical laminae are also revealcd by a varicty of markers, including cytochrome oxidase, 2-deoxyglucose, and molecular markers of synaptic density such as antibodies to synaptic vesicle proteins (Chun and Shatz, 1988; Borowsky and Collins, 1989).

One can imagine two general ways in which these several features of cortical organization might be related to increased vascularization. One possibility is that the neuropil of cortical modules is structurally similar to the adjacent neuropil, but inherently more active. In this view, neurons participating in blobs or stripes, for example, would support more action potentials per unit time than would their counterparts in adjacent regions. This possibility is given some credibility by the fact that cells within blobs respond to visual stimuli of all orientations (Livingstone and Hubel, 1984; Hubel and Livingstone, 1987). Thus, most natural stimuli would be expected to activate blob neurons; in contrast, neurons in interblob regions are selective for lines of particular orientations and would thus be activated by only a subset of visual stimuli. The putatively greater electrical activity of blobs or other modules might then stimulate increased vascularization.

A second possibility is that the neurons in blob and interblob regions, or stripe and interstripe regions, are equally active, and that such modules differ from the adjacent cortex primarily by virtue of greater neuropil density. This possibility is also supported by several of the staining properties mentioned above;
Table 4. Measurements of the overall microvessel length per unit area in standard $1.0-\mathrm{mm}$-wide strips of cortex from area 17 and area 18 at the area 17/18 border (see Fig. 6)

\begin{tabular}{clll} 
& \multicolumn{2}{l}{ Length/area $\left(\mathrm{mm} / \mathrm{mm}^{2}\right)$} & \\
\cline { 2 - 3 } Sample & Area 17 & Area 18 & \\
Difference
\end{tabular}

The average difference observed in vessel density between the primary and secondary visual cortex for the entire set of samples $(26 \%)$ is highly significant $(p<0.001$, independent Student's $t$ test $)$. Percent difference $=[($ area 17 value area $18 \mathrm{value}) /$ area 18 value] $\times 100$. 

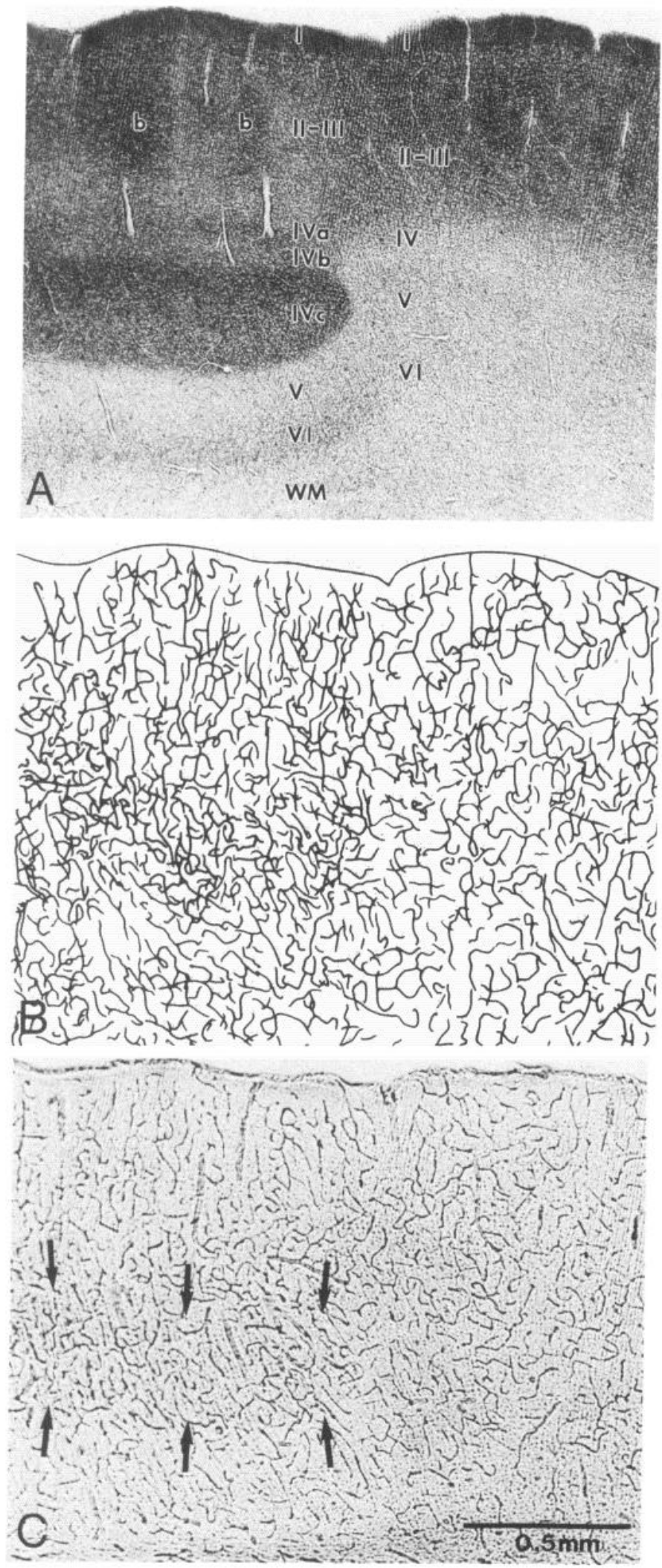

Figure 6. Greater overall vascularization of the primary compared to the secondary visual cortex. $A$, Cytochrome oxidase-stained coronal section showing $1.0-\mathrm{mm}$-wide strips of area 17 and area 18 at the area $17 / 18$ border. $W M$, white matter, $b$, blobs. $B$, Camera lucida tracing of microvessels in the same field as in $A$. C, Adjacent unstained section showing that the area 17/18 border can be discerned from the different distribution of microvessels in these two cytoarchitectonic areas. Arrows for example, the greater reactivity of blobs to cytochrome oxidase and neurotransmitter markers is in accord with this idea. In this interpretation, the increased vascularity of blobs and other modules would arise because of their physical structure (the density of their neuropil), rather than from a greater rate of electrical activity within structurally equivalent regions of cortical neuropil. At the present time, we have no basis for deciding between these possibilities (which are, in any event, not mutually exclusive).

Another implication of our analysis of blood vessels in the primate visual cortex concerns noninvasive imaging. Intravascular contrast agents have been used for several decades to reveal patterns of large blood vessels and associated pathologies in the human brain (Taveras and Wood, 1976). The smallest vessels imaged in clinical settings are of the order of $0.2 \mathrm{~mm}$ in diameter or greater; such vessels are therefore much larger than those examined here. Novel techniques of digital subtraction radiography (Kruger and Riederer, 1984) or magnetic resonance microscopy (Eccles and Callaghan, 1986; Johnson et al., 1986) may soon make it possible to see microvessel distribution in living subjects at the level of organization that we have described. This would, in principle, allow cortical modules such as blobs or stripes, and features such as the area $17 / 18$ border, to be imaged in vivo. Such imaging, if carried out on multiple occasions, would permit a noninvasive assessment of changes in the number, size, and arrangement of modules in the developing cortex (see Purves and LaMantia, 1990).

In summary, the distribution of cortical microvessels in the monkey visual cortex reflects the pattern of iterated cortical modules such as blobs and stripes, and of cytoarchitectonic features such as laminae and areal boundaries. The increased vascularization of glomeruli in the rat olfactory bulb (Borowsky and Collins, 1989) and of barrels in the rat somatosensory cortex (Patel, 1983) suggests that this relationship is a general one. A rationale for the increased vascularity of such units may be that each of these entities represents a focus of neuropil with greater metabolic and/or electrical activity than the surrounding neural tissue. Whatever the explanation, this relationship raises the possibility of noninvasively imaging the modular, laminar, and areal architecture of the living brain by taking advantage of underlying microvessel patterns.

\section{References}

Borowsky IW, Collins RC (1989) Metabolic anatomy of brain: a comparison of regional capillary density, glucose metabolism, and enzyme activities. J Comp Neurol 288:401-413.

Carroll EW, Wong-Riley MTT (1984) Quantitative light and electron microscopic analysis of cytochrome oxidase-rich zones in the striate cortex of the squirrel monkey. J Comp Neurol 222:1-17.

Chun JJM, Shatz CJ (1988) Redistribution of synaptic vesicle antigens is correlated with the disappearance of a transient synaptic zone in the developing cerebral cortex. Neuron 1:297-310.

Eccles CD, Callaghan PT (1986) High-resolution imaging. The NMR microscope. J Magn Reson 68:293-298.

Fitzpatrick D, Lund JS, Schmechel DE, Towles AC (1987) Distribution of GABAergic neurons and axon terminals in the macaque striate cortex. J Comp Neurol 264:73-91.

indicate layer IVc. The overall microvessel density in area 17 of this sample was $30.8 \mathrm{~mm} / \mathrm{mm}^{2}$, and in area $18,24.0 \mathrm{~mm} / \mathrm{mm}^{2}$. This difference between the two cytoarchitectonic regions $(28 \%)$ is similar to the average difference $(26 \%)$ in 20 such samples examined (see Table 4). 
Hendrickson AE (1985) Dots, stripes, and columns in monkey visual cortex. Trends Neurosci 8:406-410.

Hendrickson AE, Hunt SP, Wu J-Y (1981) Immunocytochemical localization of glutamic acid decarboxylase in monkey striate cortex. Nature 292:605-607.

Hendry SHC, Jones EG, Hockfield S, McKay RDG (1988) Neuronal populations stained with the monoclonal antibody CAT-301 in the mammalian cerebral cortex and thalamus. J Neurosci 8:518-542.

Horton JC (1984) Cytochrome oxidase patches: a new cytoarchitectonic feature of monkey visual cortex. Philos Trans R Soc Lond [Biol] 304:199-253.

Horton JC, Hubel DH (1981) Regular patchy distribution of cytochrome oxidase staining in primary visual cortex of macaque monkey. Nature 292:762-764.

Hubel DH (1986) Blobs and color vision. Cell Biophys 9:91-102.

Hubel DH (1988) Eye, brain, and vision. Scientific American Library Series. New York: Freeman.

Hubel DH, Livingstone MS (1987) Segregation of form, color, and stereopsis in primate area 18. J Neurosci 7:3378-3415.

Humphrey AL, Hendrickson AE (1983) Background and stimulusinduced patterns of high metabolic activity in the visual cortex (area 17) of the squirrel and macaque monkey. J Neurosci 3:345-358.
Johnson GA, Thompson MB, Gewalt SL, Hayes CE (1986) Nuclear magnetic resonance imaging at microscopic resolution. J Magn Reson 68:129-137.

Kruger RA, Riederer SJ (1984) Basic concepts of digital subtraction angiography. Boston: Hall.

Livingstone MS, Hubel DH (1984) Anatomy and physiology of a color system in the primate visual cortex. J Neurosci 4:309-359.

Livingstone MS, Hubel DH (1987) Connections between layer 4B of area 17 and the thick cytochrome oxidase strips of area 18 in the squirrel monkey. J Neurosci 7:3371-3377.

Patel U (1983) Non-random distribution of blood vessels in the posterior region of the rat somatosensory cortex. Brain Res 289:65-70.

Purves D, LaMantia A-S (1990) Numbers of "blobs" in the primary visual cortex of neonatal and adult monkeys. Proc Natl Acad Sci USA $87: 5764-5767$.

Taveras JM, Wood EH (1976) Diagnostic neuroradiology, 2d ed. Baltimore: Williams and Wilkins.

Van Essen DE (1979) Visual areas of the mammalian cerebral cortex. Annu Rev Neurosci 2:227-263.

Wong-Riley MTT (1979) Changes in the visual system of monocularly sutured or enucleated cats demonstrable with cytochrome oxidase histochemistry. Brain Res 171:11-28. 